\title{
A single-lithography SOI rib waveguide sensing circuit with apodized low back-reflection surface grating fiber coupling
}

\author{
Valentin J. Dubois, Mikael Antelius, Hans Sohlström, Kristinn B. Gylfason \\ KTH - Royal Institute of Technology, School of Electrical Engineering, \\ Microsystem Technology Lab, Osquldas väg 10, SE-100 44 Stockholm, Sweden
}

\begin{abstract}
We present a single-lithography Mach-Zehnder interferometer sensor circuit, with integrated low back-reflection input and output grating couplers. The low back-reflection is accomplished by a duty cycle apodization optimized for coupling light between single-mode silica fibers and the nanometric silicon-on-insulator (SOI) rib-waveguides. We discuss the design, fabrication, and characterization of the circuit. The apodization profile of the gratings is algorithmically generated using eigenmode expansion based simulations and the integrated waveguides, splitters, and combiners are designed using finite element simulations. The maximum simulated coupling efficiencies of the gratings are $70 \%$ and the multimode interference splitters and combiners have a footprint of only $19.2 \times 4.5 \mu \mathrm{m}^{2}$. The devices are fabricated on an SOI wafer with a $220 \mathrm{~nm}$ device layer and $2 \mu \mathrm{m}$ buried oxide, by a single electron beam lithography and plasma etching. We characterize the devices in the wavelength range from 1460-1580 $\mathrm{nm}$ and show a grating pass-band ripple of only $0.06 \mathrm{~dB}$ and grating coupling efficiency of $40 \%$ at $1530 \mathrm{~nm}$. The integrated Mach-Zehnder interferometer has an extinction ratio of $-18 \mathrm{~dB}$ at $1530 \mathrm{~nm}$ and between -13 and -19 $\mathrm{dB}$ over the whole $1460-1580 \mathrm{~nm}$ range.
\end{abstract}

Keywords: Integrated Mach-Zehnder interferometer, SOI rib waveguide, single-mode criteria, apodized surface grating coupler, multimode interference coupler

\section{INTRODUCTION}

Integrated silicon-on-insulator (SOI) Mach-Zehnder interferometers (MZIs) are used as modulators, ${ }^{1,2}$ wavelength multiplexers, ${ }^{3,4}$ and more recently as sensors. ${ }^{5,6}$ In all these applications, proper design of each optical component constituting the MZI is essential to reach the best performance. High extinction ratios, low parasitic reflections, low wavelength dependence, and low optical losses are the most important aspects to consider. For sensing, the two first have a predominant role as they directly impact the detection limit. In addition, a compact design along with a simple fabrication is important for commercialization.

With the combined improvements achieved recently in simulation and fabrication, it has become possible to fabricate integrated optical structures working in good agreement with simulation models. Most difficulties now lie in identifying and evaluating the trade-offs to be made for a specific application. For example, lower optical losses in waveguides are achieved with a rib configuration, since light is less subject to sidewall scattering, but this is done at the expense of circuit compactness.

Surface grating couplers have proven efficient for coupling light between single-mode silica fibers and nanometric silicon waveguides. However, the inclusion of grating couplers usually adds lithography steps to photonic circuit fabrication. For rapid prototyping, and cost sensitive applications like disposable sensors, a simple fabrication is a key enabler. Furthermore, in high index contrast systems like SOI, back-reflections into the photonic circuit can produce unacceptable interferences.

In this work, we present the design, fabrication, and characterization of an integrated Mach-Zehnder interferometer circuit for sensing which combines high extinction ratio, low parasitic signals, and compactness with a single E-beam lithography and plasma etch fabrication sequence. The simulation study includes the design and

Further author information: (Send correspondence to K.B.G.)

K.B.G.: E-mail: kristinn.gylfason@ee.kth.se, Telephone: +46 87909231

V.J.D.: E-mail: dubios@kth.se, Telephone: +46 87906285

Silicon Photonics and Photonic Integrated Circuits III, edited by Laurent Vivien, Seppo K. Honkanen, Lorenzo Pavesi, Stefano Pelli, Proc. of SPIE Vol. 8431, 84311Q - @ 2012 SPIE · CCC code: 0277-786X/12/\$18 · doi: 10.1117/12.921815 


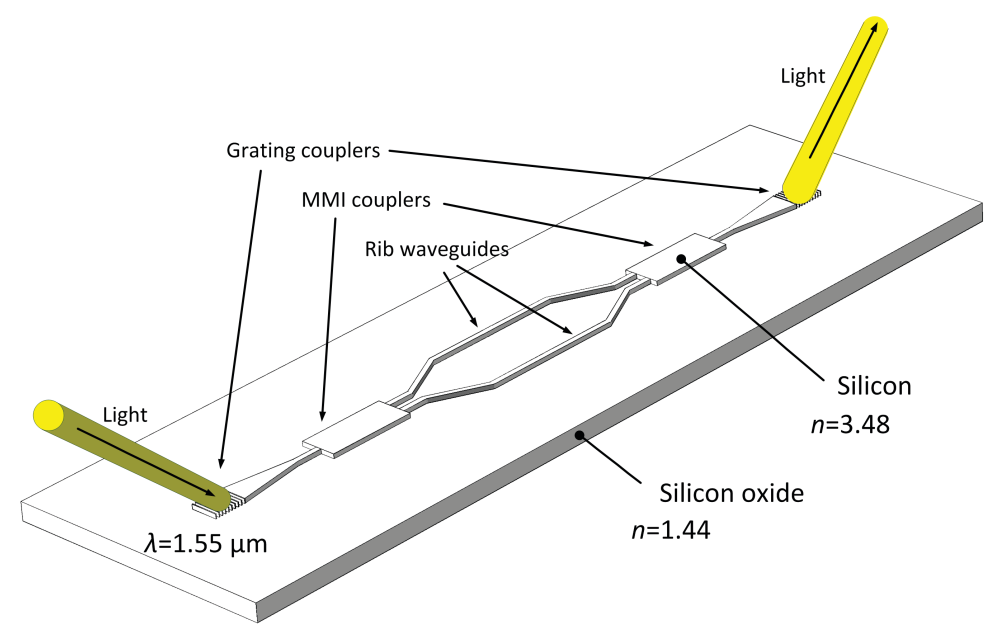

Figure 1. A schematic of the rib waveguide based sensor chip elaborated in this work. Light is coupled into and out of the chip by low back-reflection surface grating couplers, and split and combined on-chip by multimode interference couplers (MMIs).

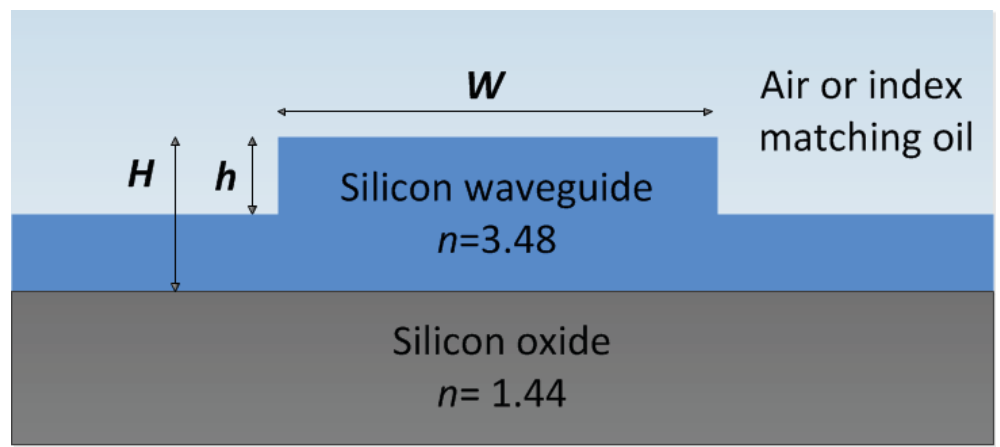

Figure 2. A cross-section of the rib waveguide. In this work, the thickness of the silicon layer is $H=220 \mathrm{~nm}$. For low loss single-mode propagation, the etch depth of the rib is chosen $h=120 \mathrm{~nm}$ and the width of the rib $W=450 \mathrm{~nm}$.

optimization of small cross-section single-mode rib waveguides, compact low wavelength dependence multimode interference couplers (MMI), and high efficiency apodized grating couplers (GC). Fig. 1 is a schematic overview of the device. The working wavelength is $\lambda=1.55 \mu \mathrm{m}$

\section{DESIGN}

\subsection{Single-mode rib waveguides}

The fundamental optical structure of an integrated MZI is the waveguide. A waveguide consisting of a slab with a strip, as shown in the cross-section in Fig. 2, is called a rib waveguide. Two important consequences, when compared to strip waveguides, can be noted: the single-mode criteria is affected by the lower confinement of light at the vertical edges of the waveguide, and the scattering losses are decreased due to the smaller overlap of the propagating wave with the roughness of the etched sidewalls. Single mode devices are preferred for sensing, since read-out is much simplified compared to multimode devices. Semi-empiric single-mode criterion formulas have been given for the case of rectangular rib waveguides. ${ }^{7}$ They are, however, not valid for thin devices. Some other works have therefore focused on finding formulas valid for smaller rib waveguides, ${ }^{8}$ but none were found for dimensions close to those of our design. We thus base our design on simulations.

As shown in Fig. 2, with the refractive indexes fixed, three parameters affect the design: the height of the waveguide $H$, the etch depth $h$, and the waveguide width $W$. The waveguide is manufactured on a $220 \mathrm{~nm}$ device layer SOI wafer, following the standard used at the ePIXfab silicon photonics foundries, and thus the height $H$ of the waveguide is fixed. The Comsol Multiphysics ${ }^{\circledR}$ simulation package provides a vectoral finite element mode 


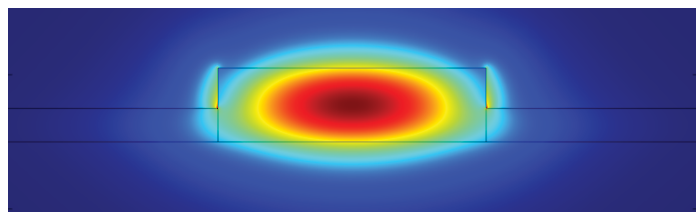

(a)

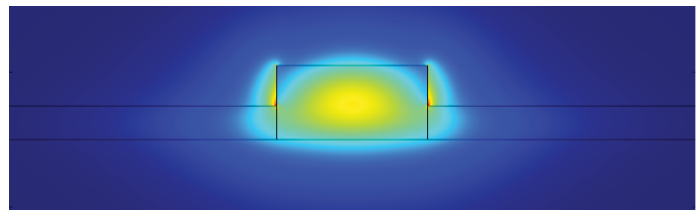

(c)

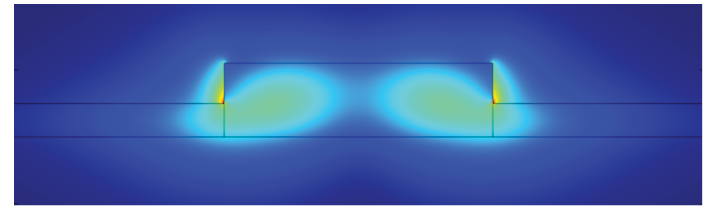

(b)

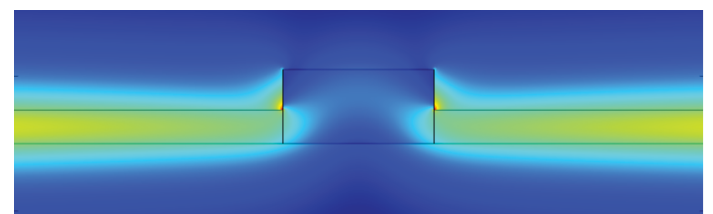

(d)

Figure 3. Cross sections of rib waveguides with $H=220 \mathrm{~nm}$ and $h=120 \mathrm{~nm}$, showing the distribution of the TE mode electric field norm of the zeroth and first order modes, as calculated with Comsol Multiphysics ${ }^{\circledR}$ at $\lambda=1.55 \mu \mathrm{m}$. The simulation windows are bigger than shown. (a) and (b) $W=800 \mathrm{~nm}$, (c) and (d) $W=450 \mathrm{~nm}$.

solver suitable for the optimization of the other parameters. We carried out a waveguide mode analysis in order to reveal the single-mode criteria of $220 \mathrm{~nm}$ thick rib waveguide.

Planar waveguide devices support two polarizations, both of which can be used for sensing. Here, we focus exclusively on the TE polarization, since a number of novel sensing devices that aim to increase the overlap of light and sample, such as slot-waveguide and photonic crystal waveguide based devices, use this polarization.

The mode confinement is defined as the fraction of the optical power of the mode propagating inside the strip part of the waveguide, including the slab region lying under it. To ensure single-mode propagation in a rib waveguide of a given etch depth $h$, the cutoff width of the first order mode needs to be determined. However, our simulations show that there is a continuous change of the confinement of the first order mode, as the waveguide width decreases and the confinement approaches zero. The mode will propagate more and more in the outer slab region, and less and less in the strip part of the waveguide (see Figs. 3(b) and 3(d)). In other words, the effective index of the first order mode approaches that of the slab. At the limit, the transverse expansion of the mode gets very large and the majority of the mode occupies the slab region. The precision wanted for the cutoff width mainly depends on how wide the simulation model is made. ${ }^{9}$

In the selection of $W$ and $h$, an intrinsic trade-off exists between the low parasitic signals and compactness. On one hand, high confinement of the light is required to achieve sharp bends with low loss ${ }^{10}$ and small multimode interference couplers for a compact device. On the other hand, high refractive index contrast increases Fresnel reflections which yield parasitic interferences. Since light scattering due to sidewall roughness cannot easily be modeled to quantify the waveguide losses, a qualitative compromise has been chosen with $h=120 \mathrm{~nm}$.

The etching depth $h$ being fixed, only the width $W$ remains to be chosen. In contrast to $h$, the width $W$ can easily be chosen from simulations: $W$ is the largest width yielding a single-mode rib waveguide with $h=120 \mathrm{~nm}$. Fig. 4 shows the plot of the confinement of the zeroth and first order modes as function of $W$ and suggests that below $450 \mathrm{~nm}$ the first order mode is not bound to the rib waveguide. We therefore decided to fabricate $450 \mathrm{~nm}$ wide rib waveguides, in order to only support the zeroth order TE mode.

By varying the wavelength in the simulations, we can extract the expected value of the group index of the zeroth order TE mode propagating in the final waveguide design

$$
n_{\mathrm{g}}=n_{\mathrm{e}}-\lambda \frac{\partial n_{\mathrm{e}}}{\partial \lambda}=2.635+1.5 \times 10^{-6} \cdot 7.3 \times 10^{5}=3.7,
$$

where $n_{\mathrm{e}}$ is the mode effective index at $\lambda=1.5$.

\subsection{Multimode interference coupler}

Multimode interference couplers are based on the physical principle of self-imaging. ${ }^{11}$ Due to reflections on the inner walls of a multimode cavity exited by a single-mode waveguide, regions where photons are concentrated and 


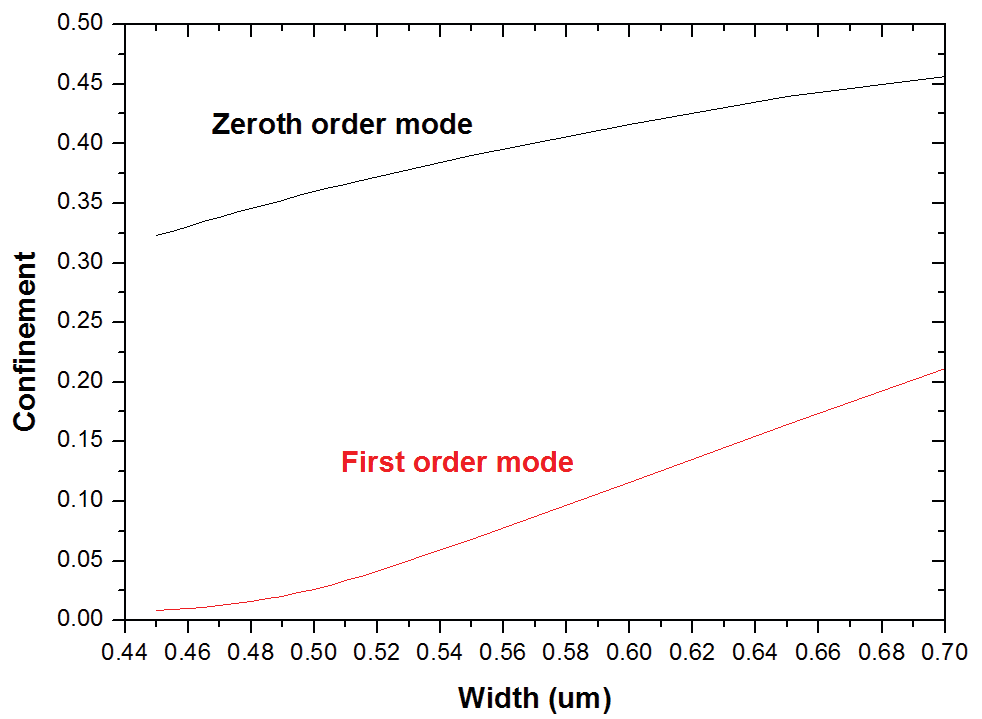

Figure 4. The fraction of the TE mode power propagating inside the rib as function of the waveguide width $W$, for $H=220 \mathrm{~nm}$ and $h=120 \mathrm{~nm}$.

regions where no photons pass through appear, as a result of wave interference. The size of the cavity is adjusted to the splitting or combining ratio wanted, which can be theoretically varied from two to tens. Furthermore, wavelength dependence and Fresnel reflections at waveguide discontinuities must be taken into account in the design of waveguide splitters and combiners.

In the design of an MMI splitter, the following aspects must be considered:

- The type of excitation. For a single-mode input, the intensity pattern is specific to that of the zeroth order mode. Each mode having a unique pattern, the picture that results from a multimode excitation is the interference of all these patterns.

- The wavelength. If the wavelength is shifted, the whole intensity pattern inside the cavity moves and the self-images are not focused in the output branches anymore.

- The effective refractive indexes. In order to simplify the simulations, only 2D models were used. The effective index method was applied to our rib configuration and gave $n_{\text {eff }}=2.869$ and $n_{\text {eff }}=2.204$, inside and outside of the cavity of the MMI, respectively, with $H=220$ and $h=120 \mathrm{~nm}$ as fixed in section 2.1 .

- The power balance. To achieve high extinction ratio in the MZI, power balance is required between the arms. For a $1 \times 2$ splitter, power is equally divided with a symmetric structure.

- The coupling between the branches. When the self-images are close, directional coupling between the output branches can occur.

In a $1 \times 2$ splitter, these parameters are easily optimized without significant trade-offs. Only the wavelength dependence has required the addition of input and output tapers to encompass larger sections of light. In addition to reducing the wavelength dependence, the tapers also relax the tolerances of the etch depth and decrease the Fresnel reflections at the transitions.

Due to the reciprocity of our system, once a working splitter is designed, a combiner is obtained by exciting the outputs instead of the input. Fig. 5 presents a simulation of the optimized MMI splitter used in this work, at $\lambda=1.55 \mu \mathrm{m}$. The color scale indicates the time averaged optical power flow. The width and length of the splitter cavity are $4.5 \mu \mathrm{m}$ and $19.3 \mu \mathrm{m}$, respectively. The length of the linear tapers is $10 \mu \mathrm{m}$, and the tapering angle is $1.9^{\circ}$. The separation of the output branches is $1.9 \mu \mathrm{m}$. 


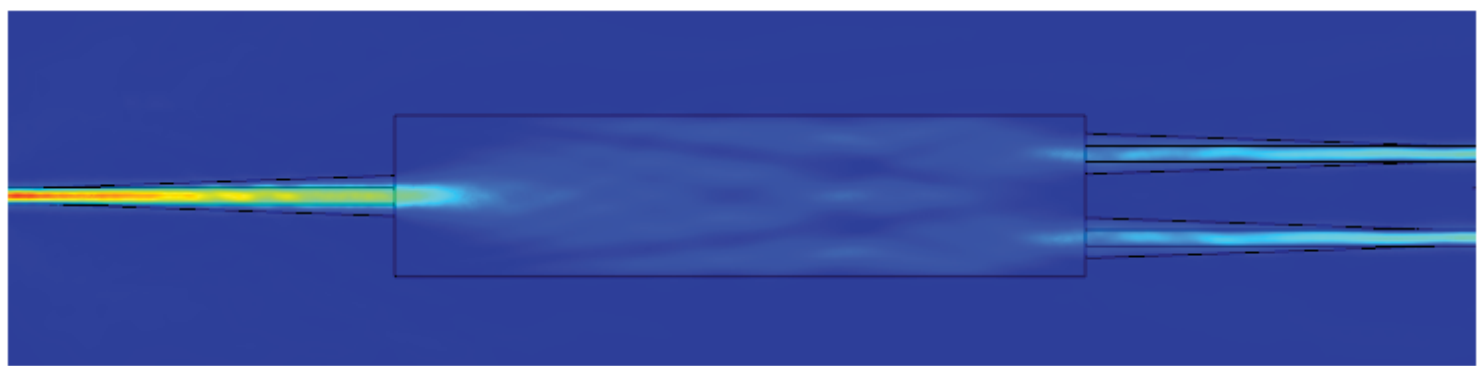

Figure 5. A top view of the optimized multimode interference splitter used in this work, as simulated with Comsol Multiphysics ${ }^{\circledR}$. The color scale indicates the time averaged optical power flow at $\lambda=1.55 \mu \mathrm{m}$. The width and length of the splitter cavity are $4.5 \mu \mathrm{m}$ and $19.3 \mu \mathrm{m}$, respectively. The length of the linear tapers is $10 \mu \mathrm{m}$, and the tapering angle is $1.9^{\circ}$. The separation of the output branches is $1.9 \mu \mathrm{m}$.

\subsection{Apodized grating coupler}

The small mode dimensions of single-mode SOI waveguides makes fiber coupling to on-chip waveguides challenging. The mode overlap between the on-chip waveguide and the fiber is very low, since the mode diameter of the single-mode fiber is much larger than that of the integrated waveguide. This results in a low coupling efficiency, and sub-micrometer positioning requirement for alignment to the on-chip waveguide.

Using surface grating couplers, the mode matching problem can be solved by expanding the width of the on-chip silicon waveguide, and etching a grating into the expanded section that diffracts light out of plane into a fiber placed normal to the surface. This increases coupling efficiency and relaxes alignment tolerances. A coupling efficiency of $37 \%$ has been reported for periodic shallow-etched SOI grating couplers. ${ }^{12}$ By applying reflectors below the gratings to reduce the loss to the substrate, coupling efficiencies of up to $69.5 \%$ have been measured, ${ }^{13}$ but this approach adds significantly to fabrication complexity and requires the use of non-standard substrates.

For sensing, apart from high coupling efficiency, a low back-reflection is required. Light that reflects back and forth between the grating couplers will create parasitic interferences in the chip. A shallow grating etch can be employed to reduce this effect, but at the cost of poorer matching to the fiber mode. Here, we employ a grating with a $120 \mathrm{~nm}$ etch into the $220 \mathrm{~nm}$ device layer and a fill factor apodization, ${ }^{14}$ i.e. we algorithmically optimize each gap and bar in the grating. The main benefits of this approach are: a low back-reflection into the silicon waveguide, the use of standard substrates, and a single required lithography step for the whole rib waveguide based sensing circuit. We have previously designed and evaluated a through-etched apodized grating on the same type of SOI substrate as used here. ${ }^{15}$

The gratings were designed for operation at $\lambda=1.55 \mu \mathrm{m}$, using a refractive index of 3.497 for silicon and 1.444 for silica. For simulating and optimizing the grating coupler performance we used the eigenmode expansion technique with perfectly matched boundary layers, as implemented in the open-source software package CAMFR. ${ }^{16}$ The design starts with an exhaustive search for the periodic grating yielding the highest coupling efficiency to a single-mode silica fiber tilted at $10^{\circ}$, in the parameter space of grating fill factor between 0 and 1 and period between 200 and $1500 \mathrm{~nm}$. The best result was found for a grating with a fill factor of 0.75 and a period of $640 \mathrm{~nm}$ (i.e. gaps of $160 \mathrm{~nm}$ and bars of $480 \mathrm{~nm}$ ). This grating has a coupling efficiency of $-2.2 \mathrm{~dB}$ $(61 \%)$ to the fiber, but a back-reflection of $-13 \mathrm{~dB}$. Using an algorithm to vary the width of each gap and bar, ${ }^{14}$ the coupling efficiency was increased to $-1.5 \mathrm{~dB}(70 \%)$, and the back-reflection reduced to $-33 \mathrm{~dB}$. Table 1 shows the design of the optimal grating, and Fig. 6(a) shows the electric field $\left(E_{y}\right)$ distribution in a cross-section of the grating in the $\mathrm{x}-\mathrm{z}$ plane.

Fig. 6(b) shows a contour plot of the simulated coupling efficiency as a function of wavelength and fiber placement along the $\mathrm{z}$ axis. The bars of the optimized grating are plotted on the $\mathrm{z}$ axis in the background. From the image we see that the the optimum coupling of $71 \%$ occurs at a wavelength of $1553 \mathrm{~nm}$ and with the fiber placed $5 \mu \mathrm{m}$ into the grating. Furthermore, the grating has a $3 \mathrm{~dB}$ bandwidth of $70 \mathrm{~nm}$ and $3 \mathrm{~dB}$ fiber placement tolerance of $7.5 \mu \mathrm{m}$ along the grating. 
Table 1. The dimensions of the apodized grating design with 20 cells. The grating is optimized for transmission at $\lambda=1.55 \mu \mathrm{m}$, and for fabrication on an SOI substrate using a single $120 \mathrm{~nm}$ etch into a $220 \mathrm{~nm}$ device layer on top of $2 \mu \mathrm{m}$ buried oxide.

\begin{tabular}{|c|c|c|c|c|c|c|c|c|c|c|c|c|c|c|c|c|c|c|c|c|}
\hline Cell & 1 & 2 & 3 & 4 & 5 & 6 & 7 & 8 & 9 & 10 & 11 & 12 & 13 & 14 & 15 & 16 & 17 & 18 & 19 & 20 \\
\hline $\mathrm{Ga}$ & 130 & 130 & 130 & 130 & 140 & 160 & 170 & 160 & 180 & 180 & 190 & 210 & 210 & 190 & 200 & 200 & 130 & 170 & 130 & 150 \\
\hline $\operatorname{Bar}[\mathrm{nm}]$ & 550 & 500 & 500 & 510 & 480 & 480 & 480 & 470 & 470 & 470 & 450 & 440 & 440 & 440 & 460 & 430 & 450 & 440 & 420 & 540 \\
\hline
\end{tabular}

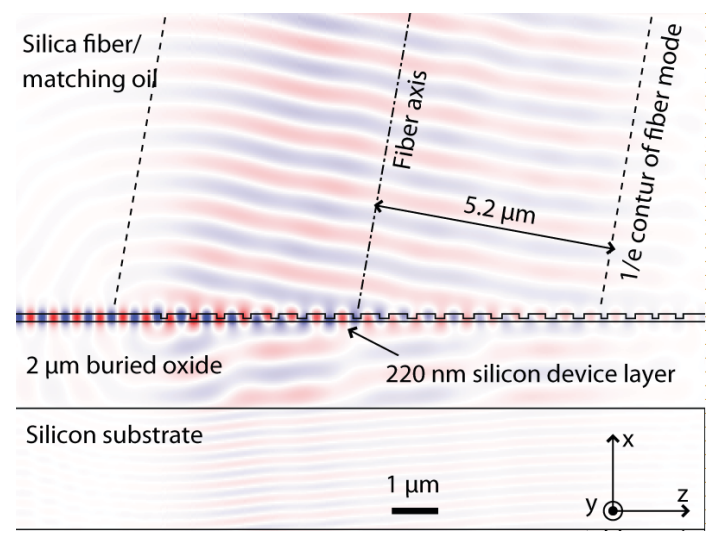

(a)

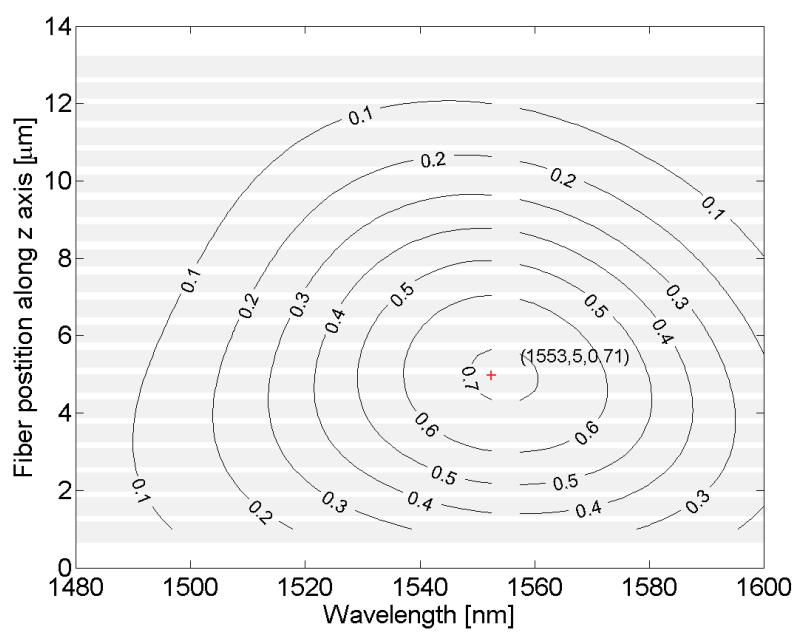

(b)

Figure 6. (a) The simulated $E_{y}$ field distribution in a cross-section along the waveguide axis, for the TE mode propagating from the single-mode silicon waveguide on the left and coupling into a single-mode fiber at a $10^{\circ}$ angle to the surface normal. (b) A contour plot of the simulated coupling efficiency from the apodized grating into the single-mode fiber, as a function of wavelength and fiber placement along the $\mathrm{z}$ axis. The bars of the optimized grating are plotted on the $\mathrm{z}$ axis in the background.

\section{FABRICATION}

\subsection{Fabrication sequence}

Fig. 7 presents the main fabrication steps from the naked SOI chip to the finalized sensor chip. First, the SOI chip was cleaned in a fresh mixture of sulfuric acid $\left(\mathrm{H}_{2} \mathrm{SO}_{4}\right)$ and hydrogen peroxide $\left(\mathrm{H}_{2} \mathrm{O}_{2}\right)$. Considering the structure of the device with narrow optical waveguides, a negative resist ( $2 \%$ hydrogen silsesquioxane (HSQ), XR-1541, Dow Corning, USA) was used. The resist was spun at $2000 \mathrm{rpm}$ for $30 \mathrm{~s}$ and baked on a hotplate for $2 \mathrm{~min}$ at $150^{\circ} \mathrm{C}$, followed by $2 \mathrm{~min}$ at $220^{\circ} \mathrm{C}$. The resist was exposed using a Raith Turnkey 150 E-beam lithography system, using a $10 \mu \mathrm{m}$ aperture and a $25 \mathrm{kV}$ acceleration voltage. The patterns were developed directly after the exposure in ma-D 532s developer (TMAH) for 2 min. To prepare the photoresist for the etching, the chip was hard baked on a hot plate at $400^{\circ} \mathrm{C}$ for $40 \mathrm{~min}$. This hardens the resist for a better resistance to the reactive ion etch that follows in a $\mathrm{Cl}_{2} / \mathrm{HBr} / \mathrm{O}_{2} / \mathrm{Ar}$ plasma. Once the targeted etch depth was obtained, the remaining photoresist was stripped in hydrofluoric acid (HF). Fig. 8 is an optical microscope image of the completed integrated Mach-Zehnder interferometer sensor circuit.

\subsection{Proximity correction of grating exposure}

The performance of the gratings, especially coupling efficiency and reflections, is very dependent on the accuracy of fabrication. Any effect which causes the fabricated devices to differ from the simulated ones should be minimized. One example is the proximity effect, which comes from the interaction between back-scattered electrons and the photoresist, and results in an overexposure of areas of high fill factor. ${ }^{17}$

To counteract this overexposure, a correction in the E-beam dose is introduced, which takes into account the back-scattered electrons. Without proximity correction, an over-exposure in the center of the structure results, 


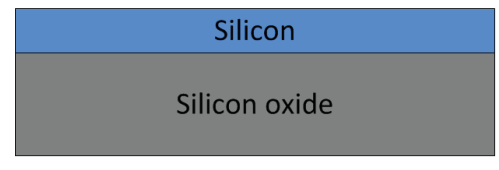

\section{SOI chip}
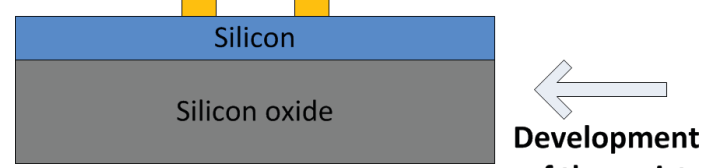

\section{of the resist}
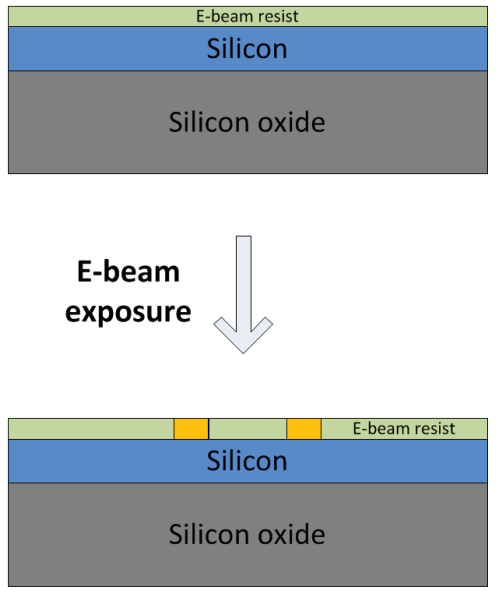

Dry etching
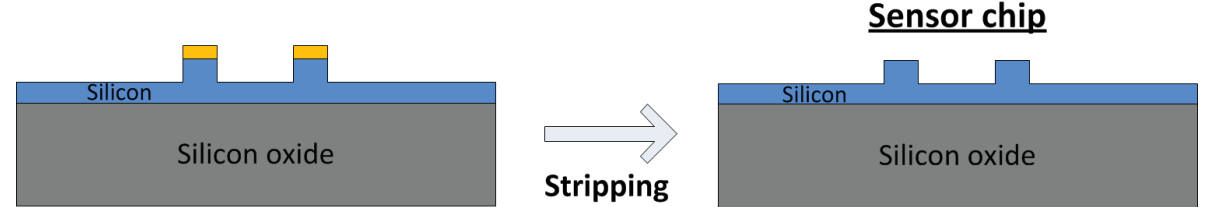

Figure 7. The fabrication sequence from the SOI chip to the sensor chip. The HSQ negative resist is spin coated onto the silicon and the narrow waveguides patterned by E-beam lithography and dry plasma etching. Finally, the remaining resist is stripped in hydrofluoric acid (HF).

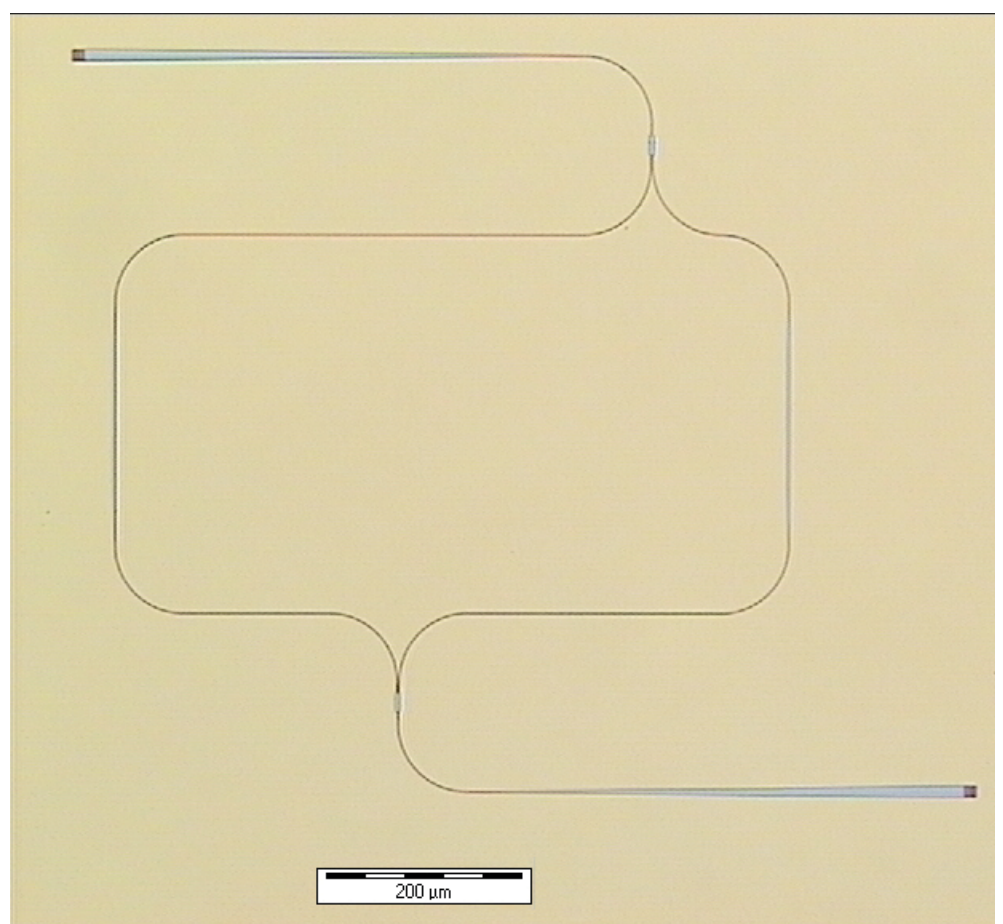

Figure 8. An optical microscope image of the complete integrated Mach-Zehnder interferometer circuit. 


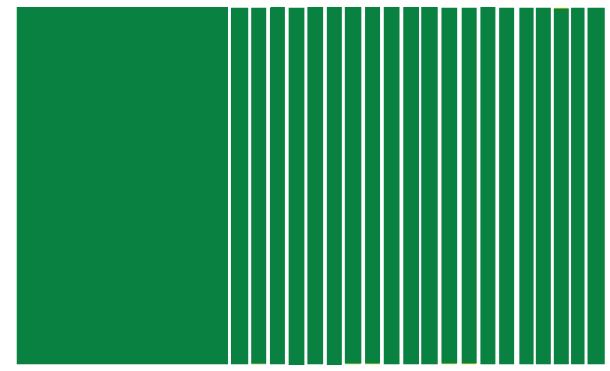

(a)

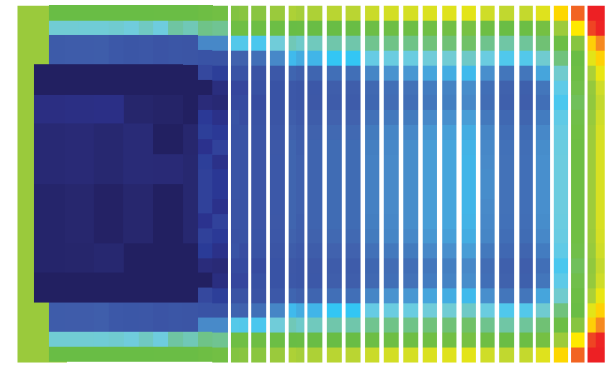

(c)

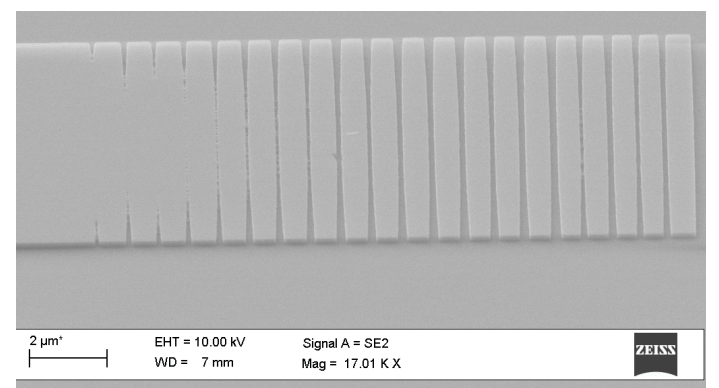

(b)

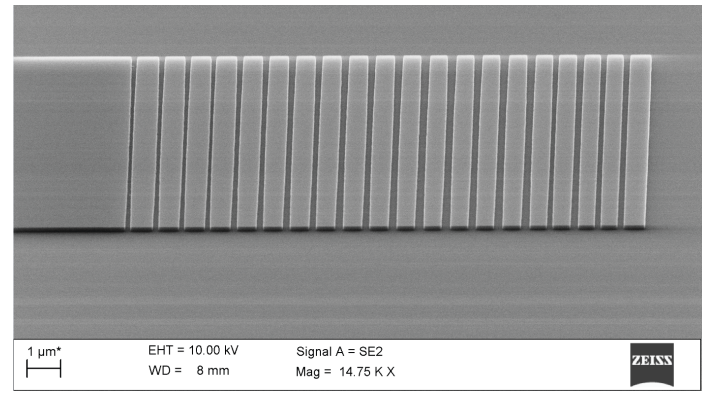

(d)

Figure 9. (a) and (b) show respectively a CAD design with dose distribution and the related SEM image of an apodized grating coupler without proximity correction; (c) and (d) same design with proximity effect dose correction. The highest dose is red at $326 \mu \mathrm{mC} / \mathrm{cm}^{2}$, the lowest is blue at $175 \mu \mathrm{mC} / \mathrm{cm}^{2}$, and green is $272 \mu \mathrm{mC} / \mathrm{cm}^{2}$

as seen in the SEM image in Fig. 9(b). The image of the gratings used in this work, shown in Fig. 9(d), confirms the reduction of the proximity effect by the use of proximity correction.

The principle of the correction is to approximate the backward-scattering with two Gaussian functions. The dose correction, $D C$, is then proportional to:

$$
D C(r) \sim \frac{1}{1+\xi}\left(\frac{1}{\alpha^{2}} e^{-\frac{r^{2}}{\alpha^{2}}}+\frac{\xi}{\beta^{2}} e^{-\frac{r^{2}}{\beta^{2}}}\right)
$$

where $r$ is the radial distance, $\xi, \alpha$ and $\beta$ are determined by the acceleration voltage of the E-beam, the type of photoresist and the material of the substrate and have been set at $0.75,14 \mathrm{~nm}$ and $2504 \mathrm{~nm}$ respectively. See ${ }^{18}$ for additional information. When the correction is applied, the grating design is broken into smaller polygons which are assigned a more suitable dose (see Fig. 9(c)). The structure should not be broken into exceedingly small polygons since the E-beam exposure time heavily depends on its number.

\section{OPTICAL CHARACTERIZATION}

\subsection{Grating couplers}

Good performance of the grating couplers is vital to be able to interrogate the MZI. In particular, high coupling efficiencies and low back-reflections are necessary. A test structure has been fabricated to evaluate the grating performance. It consists of two grating couplers and two tapers put back-to-back. For characterization, a refractive index matching oil (Cargille 50350) was used as a top cladding. This oil has a viscosity of only $19 \mathrm{cSt}$ and wets the structures with a very low contact angle, thus we assume complete filling of the grating gaps. A more durable cladding, such as a spin on glass, could be used for production devices.

The efficiency of coupling between a single mode fiber and an on-chip single mode silicon waveguide was determined using an Agilent 86082A Wavelength Domain Component Analyzer and a tunable light source with a $1 \mathrm{pm}$ line width. First, the loss of a SMF-28 patch cord was determined. The fiber was then cleaved and the two ends arranged with a $10^{\circ}$ angle and optimum coupling position in the index matching liquid over the 


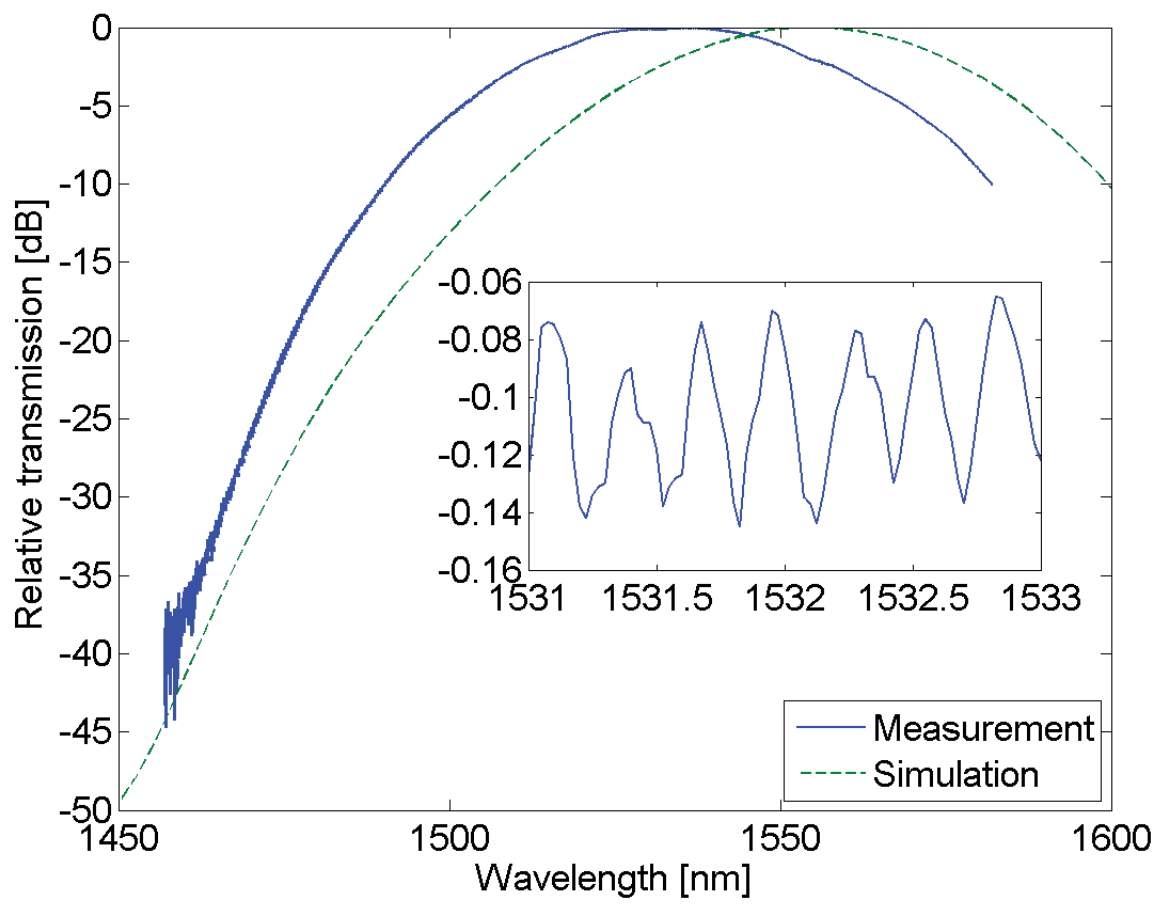

Figure 10. Relative transmission through two grating couplers back-to-back, measured with a wavelength step of 3 pm. The solid curve is the measured transmission, and the dashed curve is obtained from simulations of the optimized design using the eigenmode expansion technique. The inset is an enlargement of the experimental curve from 1531 to $1533 \mathrm{~nm}$. The pass-band ripple is $0.06 \mathrm{~dB}$.

gratings. The polarization in the input fiber was optimized for TE coupling with a "bat ear" fiber polarization controller. The excess loss through the complete structure was then measured in the wavelength range from 1460 to $1580 \mathrm{~nm}$ The coupling efficiency of each grating coupler is half of the excess loss. The best coupling efficiency observed was $40 \%$.

The measured transmission spectrum through the test structure from 1460 to $1580 \mathrm{~nm}$ is shown in Fig. 10. Also included in the image is the simulated transmission spectrum of the optimized grating design. The smooth shape of the measured curve confirms the low parasitic signals expected thanks to the apodized design and the proximity correction. The comparison of the experimental and the simulated curves shows that the measured curve is blue-shifted $19 \mathrm{~nm}$ compared to the design. Besides the shift, the shapes of the two curves are in good agreement. The shift of the spectrum is most likely due to deviations from the geometric design values during fabrication. The inset of Fig. 10 gives a closer look at the pass-band of the measured curve to evaluate the parasitic signal caused by the back-reflections. We find an average of $0.06 \mathrm{~dB}$ pass-band ripple, which is close to the recent state-of-the-art ripple of $0.02 \mathrm{~dB}$ achieved by Intel ${ }^{19}$ using a more complex design and fabrication process.

\subsection{The Mach-Zehnder interferometer}

A test structure, shown in Fig. 8, has been fabricated to extract the characteristics of the MZI. We introduce an asymmetry in the arms of the MZI, and by scanning the wavelength, we can evaluate the extinction ratio, as well as the waveguide effective group index $n_{\mathrm{g}}$, from the observed free spectral range (FSR).

The measured transmission spectrum of the MZI is shown in Figs. 11(a) and 11(b). As expected, the curve is a product of the grating curve, induced by the wavelength dependence of the grating coupling efficiency, and the MZI cosines curve, due to the light interference inside the combiner. The curve is smooth, indicating that we have the low reflections, as expected from the design of all the elements of the photonic circuit. In order to 


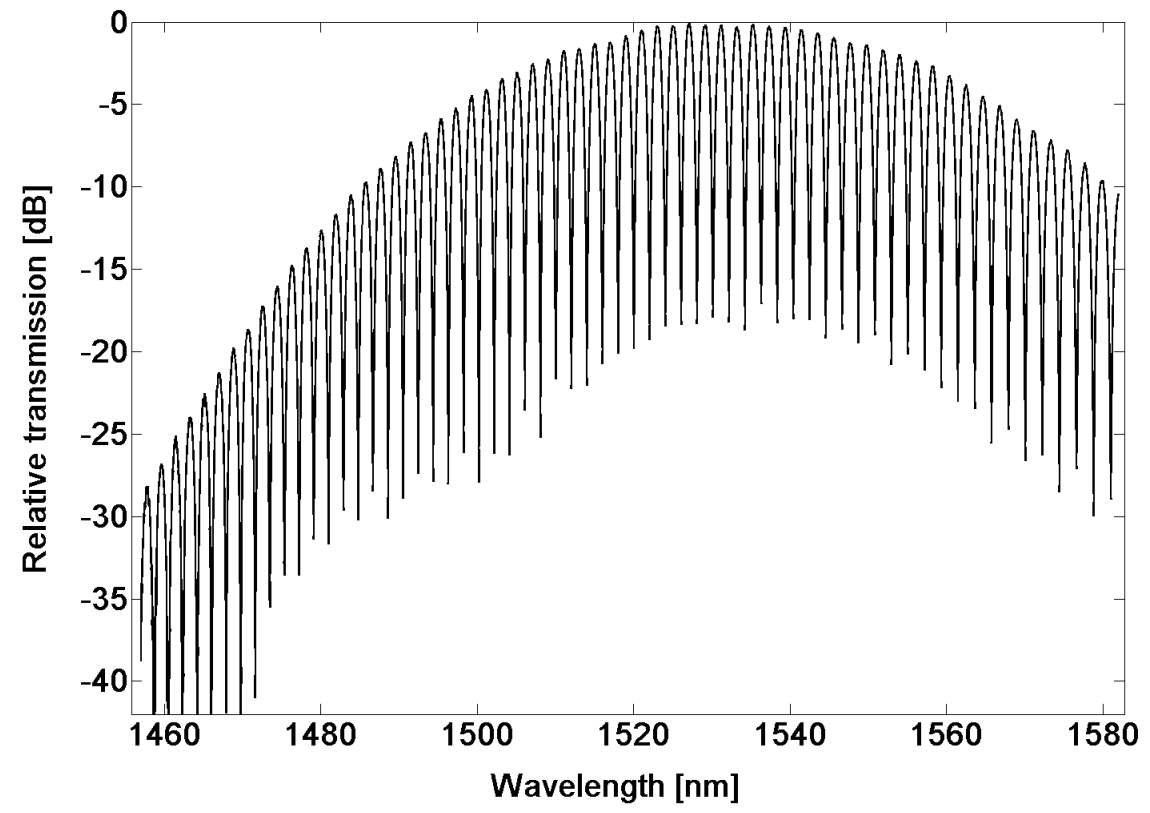

(a) The wavelength range from $1453 \mathrm{~nm}$ to $1582 \mathrm{~nm}$.

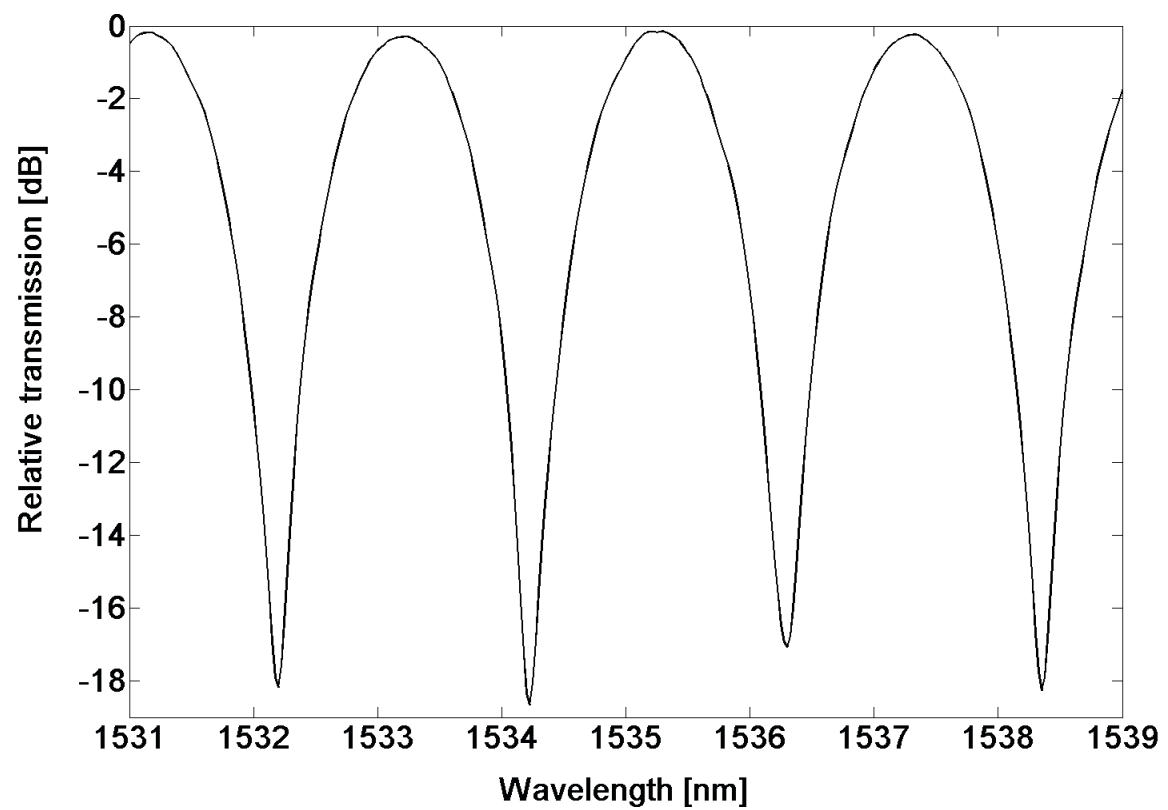

(b) An enlarged view of the wavelength range from $1531 \mathrm{~nm}$ to $1539 \mathrm{~nm}$.

Figure 11. The transmission spectrum of the complete integrated Mach-Zehnder interferometer used in this work, measured with a wavelength step of $3 \mathrm{pm}$. 
confirm the origin of the observed interferences, the silica matching oil used as top cladding has been heated up and a shift of the curve has been observed, corresponding to the change in the refractive index of the oil.

The FSR read from Fig. 11(b) is $1.9 \mathrm{~nm}$ at $\lambda=1500 \mathrm{~nm}$ and the asymmetry of the MZI arm length is $\Delta L=300 \mu \mathrm{m}$. Thus, the experimentally observed group index of the waveguide is

$$
n_{\mathrm{g}}=\frac{\lambda^{2}}{\Delta L \cdot F S R}=\frac{\left(1500 \times 10^{-9}\right)^{2}}{300 \times 10^{-6} \cdot 1.9 \times 10^{-9}}=3.9
$$

This agrees well with the group index value $n_{\mathrm{g}}=3.7$ found from the finite element mode solver simulations in section 2.1. The difference is most likely due to a deviation of the width of the waveguide during fabrication.

Another important data to extract from Fig. 11(b) is the extinction ratio, since it affects directly the detection limit of a biosensor. In Fig. 11(b), we can read $-18 \mathrm{~dB}$ at $1530 \mathrm{~nm}$ and between -13 and $-19 \mathrm{~dB}$ over the whole $1460-1580 \mathrm{~nm}$ range. The asymmetry of the MZI limits the achievable extinction ratio, since the losses in the two arms are not equal. The extinction ratio of a symmetric MZI sensor would be even lower.

\section{CONCLUSIONS}

We have presented the design and simulation of all the components of an integrated Mach-Zehnder interferometer sensor circuit, the fabrication of the sensor chip, and the optical characterization of the device.

A rib waveguide configuration was chosen to lower unwanted reflections. The dimensions of the waveguide were optimized for single-mode propagation. Tapered multimode interference couplers were chosen to split and combine light on the sensor chip for their compactness, ease of fabrication, good balance, and low wavelength dependence. The grating couplers used to couple light in and out of the chip benefited from an apodized design to decrease back-reflections and from a proximity correction for an accurate fabrication. The final design fits all the components in a single square millimeter.

We fabricated an MZI with an asymmetry of $300 \mu \mathrm{m}$ to extract its characteristics. The fabrication is done by a single E-beam exposure of a negative resist on an SOI chip, followed by a dry etching. Optical measurements were performed to characterize the grating couplers and the MZI. The results showed low reflections and high coupling efficiencies for the grating couplers, and an $-18 \mathrm{~dB}$ extinction ratio of the MZI.

\section{ACKNOWLEDGMENTS}

This work was partially supported by the Swedish Research Council under grant agreement B0460801.

\section{REFERENCES}

[1] Reed, G. T., Mashanovich, G., Gardes, F. Y., and Thomson, D. J., "Silicon optical modulators," Nature Photonics 4, 518-526 (Aug. 2010).

[2] Liu, A., Jones, R., Liao, L., Samara-Rubio, D. A., Rubin, D., Cohen, O., Nicolaescu, R., and Paniccia, M. J., "Fast silicon optical modulator," in [Photonics West 2004: Integrated Optoelectronic Devices], Proceedings of SPIE 5357, 35-44, The International Society for Optical Engineering. (July 2004).

[3] Jinguji, K., Takato, N., Hida, Y., Kitoh, T., and Kawachi, M., "Two-port optical wavelength circuits composed of cascaded Mach-Zehnder interferometers with point-symmetrical configurations," Journal of Lightwave Technology 14, 2301-2310 (Oct. 1996).

[4] Sangsiri, T., Hayee, M. I., Hoanca, B., Shieh, W., and Willner, A. E., "Stability and dynamic range of a Mach-Zehnder wavelength shifter," in [Conference on Optical Fiber Communication], 218-219, IEEE (Feb. 1997).

[5] Lechuga, L. M., Zinoviev, K., Fernández, L., Elizalde, J., Hidalgo, O. E., and Dominguez, C., "Biosensing microsystem platforms based on the integration of Si Mach-Zehnder interferometer, microfluidics and grating couplers," in [Photonics West: Integrated Optoelectronic Devices 2009], Proceedings of SPIE 7220, 72200L72200L-8, The International Society for Optical Engineering. (Feb. 2009). 
[6] Li, Y., Chen, L., Harris, E., Bao, X., and Bock, W. J., "Refractive index and temperature sensor based on double-pass in-line Mach-Zehnder interferometer," Proceedings of SPIE 7753, 77530Q-77530Q-4 (May 2011).

[7] Milosevic, M. M., Matavulj, P. S., Timotijevic, B. D., Reed, G. T., and Mashanovich, G. Z., "Design Rules for Single-Mode and Polarization-Independent Silicon-on-Insulator Rib Waveguides Using Stress Engineering," Journal of Lightwave Technology 26, 1840-1846 (July 2008).

[8] Müllner, P. and Hainberger, R., "Single-mode criterion for rib waveguides with small cross sections," in [Photonics West 2006: Integrated Optoelectronic Devices], Proceedings of SPIE 6115, 61152E-61152E-8, The International Society for Optical Engineering. (Feb. 2006).

[9] Lousteau, J., Furniss, D., Seddon, A. B., Benson, T. M., Vukovic, A., and Sewell, P., "The Single-Mode Condition for Silicon-on-Insulator Optical Rib Waveguides With Large Cross Section," Journal of Lightwave Technology 22, 1923+ (Aug. 2004).

[10] Yuanyuan, C., Qingfeng, Y., Di, Y., Shaowu, C., and Jinzhong, Y., "Structural optimizations of SOI-based single-mode rib waveguide bends [optical waveguides]," in [7th International Conference on Solid-State and Integrated Circuits Technology], 3, 2015-2017 vol.3, IEEE (Oct. 2004).

[11] Soldano, L. B. and Pennings, E. C. M., "Optical multi-mode interference devices based on self-imaging: principles and applications," Journal of Lightwave Technology 13, 615-627 (Apr. 1995).

[12] Taillaert, D., Van Laere, F., Ayre, M., Bogaerts, W., Van Thourhout, D., Bienstman, P., and Baets, R., "Grating couplers for coupling between optical fibers and nanophotonic waveguides," Japanese Journal of Applied Physics 45(8A), 6071-6077 (2006).

[13] Selvaraja, S. K., Vermeulen, D., Schaekers, M., Sleeckx, E., Bogaerts, W., Roelkens, G., Dumon, P., Van Thourhout, D., and Baets, R., "Highly efficient grating coupler between optical fiber and silicon photonic circuit," in [Conference on Lasers and Electro-Optics/International Quantum Electronics Conference], OSA Technical Digest (CD), CTuC6+, Optical Society of America (May 2009).

[14] Taillaert, D., Bienstman, P., and Baets, R., "Compact efficient broadband grating coupler for silicon-oninsulator waveguides," Optics Letters 29, 2749-2751 (Dec. 2004).

[15] Antelius, M., Gylfason, K. B., and Sohlström, H., "An apodized SOI waveguide-to-fiber surface grating coupler for single lithography silicon photonics," Optics Express 19, 3592-3598 (Feb. 2011).

[16] Bienstman, P. and Baets, R., "Optical modelling of photonic crystals and VCSELs using eigenmode expansion and perfectly matched layers," Optical and Quantum Electronics 33, 327-341 (Apr. 2001).

[17] Ren, L. and Chen, B., "Proximity effect in electron beam lithography," in [7th International Conference on Solid-State and Integrated Circuits Technology], 1, 579-582 vol.1, IEEE (Oct. 2004).

[18] Wüest, R., "An efficient proximity-effect correction method for electron-beam patterning of photonic-crystal devices," Microelectronic Engineering 67-68, 182-188 (June 2003).

[19] Na, N., Frish, H., Hsieh, I.-W., Harel, O., George, R., Barkai, A., and Rong, H., "Efficient broadband silicon-on-insulator grating coupler with low backreflection," Optics Letters 36, 2101-2103 (June 2011). 